\title{
Correlation energy functional and potential from time-dependent exact-exchange theory
}

\author{
Maria Hellgren and Ulf von Barth \\ Department of Mathematical Physics, Institute of Physics, \\ Lund University, Sölvegatan 14A, S-22362 Lund, Sweden
}

(Dated: June 21, 2018)

\begin{abstract}
In this work we have studied a new functional for the correlation energy obtained from the exactexchange (EXX) approximation within time-dependent density functional theory (TDDFT). Correlation energies have been calculated for a number of different atoms showing excellent agreement with results from more sophisticated methods. These results loose little accuracy by approximating the EXX kernel by its static value, a procedure which enormously simplifies the calculations. The correlation potential, obtained by taking the functional derivative with respect to the density, turns out to be remarkably accurate for all atoms studied. This potential has been used to calculate ionization potentials, static polarizabilities and van der Waals coefficients with results in close agreement with experiment.
\end{abstract}

PACS numbers: 31.15.Ew, 31.25.-v, 71.15.-m

\section{INTRODUCTION}

Time-dependent density functional theory (TDDFT) provides a promising and rigorous framework for treating interacting many-electron systems within a reasonable computational cost. Although the overall aim of TDDFT is to describe physical phenomena associated with excited states, strong connections between the latter and ground-state properties make it possible to obtain an improved description of the ground state through the use of TDDFT.

In many interesting cases, it is sufficient to treat systems under the influence of external perturbations weak enough to allow for a description in terms of linear response. Within this realm, the basic quantities of TDDFT are the ground-state exchange-correlation (XC) potential and the corresponding $\mathrm{XC}$ kernel $\left(f_{\mathrm{xc}}\right) .{ }^{1} \mathrm{In}$ previous work we have studied these quantities at different levels of approximation. The XC potential has been calculated for atoms at the level of the $G W$ approximation ${ }^{2}$ and the XC kernel has been presented within the exactexchange (EXX) approximation. ${ }^{3-5}$ In all these previous publications the necessary formulas have been derived from the variational formulation of many-body perturbation theory. ${ }^{6,7}$ The virtue of this approach is that the obtained results are guaranteed to obey many conservation laws and sum rules like, e.g., the $f$-sum rule, of importance to the calculated optical spectra. In the present work we have deviated from this path and instead followed an approach originally suggested by Peuckert. ${ }^{8}$

Having some approximation for the density-density response function of a many-electron system one can obtain an expression for the total ground-state energy by using the Hellmann-Feyman theorem applied to the strength of the inter-particle Coulomb interaction. ${ }^{9,10}$ And, by means of TDDFT, from any approximation for the XC potential and the corresponding $\mathrm{XC}$ kernel one can obtain an approximation to the density response function of the system. The XC part of the resulting total energy can then be differentiated once with respect to the density to yield a new approximation for the $\mathrm{XC}$ potential and then twice to yield a new approximation for the XC kernel. From these results we can obtain a new total energy which again can be differentiated to obtain a new potential and a new kernel, and so on. Of course, in practice the resulting expressions quickly become unmanageable and the proposed iterations have to be limited to one or two steps.

In previous work we have seen that the total energy obtained via the Hellman-Feynman theorem applied to the EXX approximation is very accurate giving errors of the order of $5 \%$ in the resulting correlation energies. ${ }^{3}$ These results inspired us to believe that a differentiation of the corresponding expression for the $\mathrm{XC}$ energy with respect to the density might give rise to a very accurate $\mathrm{XC}$ potential and XC kernel. And this is, indeed, what we have found in the present work, at least as far as the potential is concerned and, then, within the approximations we have been forced to do in order to obtain tractable expressions.

In the present context, the XC kernel of the EXX approximation (EXXA) has the convenient property of being linear in the Coulomb interaction allowing us to carry out the integration over the strength of the Coulomb interaction analytically. The result is a closed expression for the XC energy. This expression explicitly contains the EXX kernel $f_{\mathrm{x}}$, which give rise to numerical difficulties in the later process of differentiating the $\mathrm{XC}$ energy with respect to the density. Motivated by the fact that the EXX kernel for He is independent of the density we have neglected this density dependence for all atoms. The validity of this approximation must, of course, be verified independently but, encouraged by our excellent results, we have decided to leave this test to a future publication.

We have also employed an additional approximation which we believe to be of even less consequence. The fact that the EXX kernel is frequency dependent for all systems but for a two-electron one leads to much longer 
computational times. We have, however, seen that total energies are very insensitive to this frequency dependence and we thus recommend to neglect it. The frequency dependence will, of course, also affect the calculation of the $\mathrm{XC}$ potential except in the case of He. We have here assumed that also the potentials of the heavier atoms are relatively insensitive to this frequency dependence but we leave also the verification of this assumption to a future publication.

The XC potentials that we obtain from the first iteration of the Peuckert procedure starting from the EXXA turns out to be much better than any approximate $\mathrm{XC}$ potentials perviously obtained and they are actually very close to the exact ones ${ }^{11}$ where these are known. For a number of spherical atoms, we have used these new potentials to calculate total energies, static polarizabilities, and van der Waals coefficients using the expression for the XC kernel obtained within the EXXA. In all cases we find excellent agreement with experiment. We thus conclude that we now have an affordable and well defined way of obtaining accurate results for ground-state properties and low lying excitations of many-electron systems.

\section{CORRELATION ENERGY FUNCTIONAL}

A standard expression for the correlation energy can be obtained by introducing a fictitious Hamiltonian $H^{\lambda}$ with a scaled Coulomb interaction $\lambda v$ and a local multiplicative potential which guarantees that the density is constant for every value of the scaling parameter $\lambda$. At $\lambda=1, H^{\lambda}$ coincides with the fully interacting Hamiltonian and at $\lambda=0$ with the one of the non-interacting Kohn-Sham (KS) system. Using the Hellman-Feynman theorem one can show ${ }^{9,10,12}$

$$
E_{\mathrm{c}}=\frac{i}{2} \int_{0}^{1} d \lambda \int \frac{d \omega}{2 \pi} \operatorname{Tr}\left\{v\left[\chi^{\lambda}(\omega)-\chi_{s}(\omega)\right]\right\}
$$

where $\chi_{s}$ is the non-interacting $\mathrm{KS}$ response function and $\chi^{\lambda}$ is the scaled density response function. We have also used the short hand notation $\operatorname{Tr} f g=$ $\int d \mathbf{r} d \mathbf{r}^{\prime} f\left(\mathbf{r}, \mathbf{r}^{\prime}\right) g\left(\mathbf{r}^{\prime}, \mathbf{r}\right)$ for any two-point functions $f$ and $g$. Within TDDFT the function $\chi^{\lambda}$ reads

$$
\chi^{\lambda}=\chi_{s}+\chi_{s}\left[\lambda v+f_{\mathrm{xc}}^{\lambda}\right] \chi^{\lambda} .
$$

The scaled XC kernel $f_{\mathrm{xc}}^{\lambda}$ is a functional of the groundstate density and is defined as the functional derivative of the scaled XC potential $v_{\mathrm{xc}}^{\lambda}$ with respect to the density $n$.

The simplest approximation to Eq. (1) is the random phase approximation (RPA), obtained with $f_{\mathrm{xc}}^{\lambda}=0$. The RPA has the advantage of allowing for an analytical evaluation of the $\lambda$-integral in Eq. (1). The result is

$$
E_{\mathrm{c}}=-\frac{i}{2} \int \frac{d \omega}{2 \pi} \operatorname{Tr}\left\{\ln \left[1-v \chi_{s}\right]+v \chi_{s}\right\} .
$$

In the language of Feynman diagrams Eq. (3) is equal to an infinite summation of ring-diagrams.

The RPA correlation potential $v_{\mathrm{c}}$ is obtained as the functional derivative of Eq. (3) with respect to the density. If we let $V$ signify the total $\mathrm{KS}$ potential, $G_{s}$ the non-interacting KS Green function, $\chi_{s}=-i G_{s} G_{s}$ and the functional derivative is conveniently obtained via the chain rule

$$
\frac{\delta E_{\mathrm{c}}}{\delta n} \frac{\delta n}{\delta V}=\frac{\delta E_{\mathrm{c}}}{\delta G_{s}} \frac{\delta G_{s}}{\delta V}
$$

The result is the well-known linearized Sham-Schlüter (LSS) equation ${ }^{6,13}$

$$
\int \chi_{s}(1,2) v_{\mathrm{c}}(1) d 2=\int \Lambda(3,2 ; 1) \Sigma_{\mathrm{c}}(2,3) d 2 d 3 .
$$

Here, we have used the notation $\left(\mathbf{r}_{1}, t_{1}\right)=1$ etc. and introduced $\Lambda(3,2 ; 1)=-i G_{s}(3,1) G_{s}(1,2)$. The correlation part of the self-energy $\Sigma_{\mathrm{c}}$ in the RPA is given by

$$
\Sigma_{\mathrm{c}}=i \frac{\delta E_{\mathrm{c}}}{\delta G_{s}}=i v \chi^{\mathrm{RPA}} v G_{s}
$$

where

$$
\chi^{\mathrm{RPA}}=\chi_{s}+\chi_{s} v \chi^{\mathrm{RPA}}
$$

Thus, the $\Sigma_{\mathrm{c}}$ in the RPA coincides with the $G W$ selfenergy but evaluated at KS Green functions. For the purpose of obtaining the RPA potential Eq. (4) and Eq. (6) have to be solved self-consistently and has only been done so far for atoms ${ }^{2}$ and in bulk Si, LiF and solid Ar. ${ }^{14}$ The RPA is also sometimes called the linearized time-dependent (TD) Hartree approximation since $\chi^{\mathrm{RPA}}$ is obtained by allowing the electrons to respond only to the perturbing potential plus the induced Hartree potential. The next level of approximation is obtained by also including exchange effects, which leads to the RPAE approximation or the linearized TD Hartree Fock (TDHF) approximation. Within TDDFT the same level of approximation corresponds to the TDEXX approximation, in which the HF potential is replaced by the local EXX potential $v_{\mathrm{x}}$. The latter potential is obtained from the TD exchange version of the LSS equation which amounts to replacing, in Eq. (4), $v_{\mathrm{c}}$ by $v_{\mathrm{x}}$ and $\Sigma_{\mathrm{c}}$ by $\Sigma_{\mathrm{x}}=i v G_{s}$, i.e., the HF self-energy. A variation of that equation yields an equation for the EXX response kernel $f_{\mathrm{x}}$ :

$$
\begin{aligned}
\int \chi_{s} & (1,2) f_{\mathrm{x}}(2,3) \chi_{s}(3,4) d 2 d 3 \\
= & \int \frac{\delta \Sigma_{\mathrm{x}}(2,3)}{\delta V(4)} \Lambda(3,2 ; 1) d 2 d 3 \\
& +\int \Lambda(1,2 ; 4) \Delta(2,3) G_{s}(3,1) d 2 d 3 \\
& +\int G_{s}(1,2) \Delta(2,3) \Lambda(3,1 ; 4) d 2 d 3
\end{aligned}
$$

where $\Delta(2,3)=\Sigma_{\mathrm{x}}(2,3)-v_{\mathrm{x}}(2) \delta(2,3)$. A full analysis of this kernel has been performed recently. ${ }^{4}$ It is important 
to observe that the kernel $f_{\mathrm{x}}$ is an implicit functional of the density through the KS orbitals and their eigenvalues. From Eq. (7) we see that the potential $v_{\mathrm{x}}$ is an ingredient in the kernel $f_{\mathrm{x}}$ but, as discussed above, $v_{\mathrm{x}}$ is also an implicit functional of the density. For future reference we here note that an EXX kernel obtained for any density and used in Eq. (2) will generate an interacting response function which obeys the important $f$-sum rule. This is due to the fact that the procedure above guarantees that the kernel does not blow up at large frequencies, something that we proved in a previous publication. ${ }^{3}$ The fulfillment of the $f$-sum rule is crucial for the construction of new functionals for the correlation energy, one of which we will now derive.

We start by making the observation that the EXX kernel is linear in the explicit dependence on the Coulomb potential. Therefore, the $\lambda$-integration in Eq. (1) can, just as in the case of RPA, be carried out analytically yielding the result

$$
E_{c}=-\frac{i}{2} \int \frac{d \omega}{2 \pi} \operatorname{Tr}\left\{\frac{v}{v+f_{\mathrm{x}}} \ln \left[1-\left[v+f_{\mathrm{x}}\right] \chi_{s}\right]+v \chi_{s}\right\} .
$$

We note that $\operatorname{Tr} v \chi_{s}$ contains a singularity proportional to the Coulomb potential at the origin times the number of particles. This singularity must be cancelled by the first term in Eq. (8) which will only occur if the kernel remains finite at large frequencies, i.e., obey the sum rule.

Correlation energies obtained from this functional was recently presented ${ }^{3}$ but in a non self-consistent fashion using the EXX density for the evaluation. The results were excellent for many closed-shell atoms. A diagrammatic description of Eq. (8), up to second order, is given in Fig. 1. Many higher order terms do not have a strict diagrammatic representation but can be considered to simulate the higher order exchange diagrams.

In order to obtain the correlation potential from Eq. (8) we need to differentiate the correlation energy with respect to the density. We then encounter the problem of differentiating the EXX kernel with respect to the density, which is a rather cumbersome task. Such derivatives formally amounts to three-point correlation functions $\delta f_{\mathrm{x}}(1,2) / \delta n(3)$. For He, however, this derivative is zero since $f_{\mathrm{x}}=-\frac{1}{2} v$. Therefore, we have here chosen to see how well we can do by neglecting the density variation of the EXX kernel also for larger atoms. With this assumption the same procedure used for going from Eq. (3) to Eq. (4) now leads to an equation similar to Eq. (4) with $\Sigma_{\mathrm{c}}$ replaced by

$$
\Sigma_{\mathrm{c}}=i v \chi\left[v+f_{\mathrm{x}}\right] G_{s}
$$

where $\chi$ is the interacting response function in the TDEXX approximation (Eq. 2 at $\lambda=1$ and $f_{\mathrm{xc}}=f_{\mathrm{x}}$ ). The evaluation of the correlation energy form Eq. (8) is relatively time consuming due to the frequency dependence of the kernel $f_{\mathrm{x}} \cdot{ }^{3}$ The computational cost would be substantially reduced it the kernel could be kept at its static value $\left(f_{\mathrm{X}}\left(\mathbf{r}, \mathbf{r}^{\prime}, \omega\right) \approx f_{\mathrm{X}}\left(\mathbf{r}, \mathbf{r}^{\prime}, 0\right)\right)$ without seriously affecting the correlation energies. In Table I the

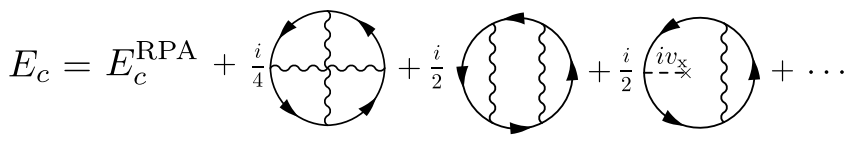

FIG. 1: With $f_{\mathrm{xc}}=f_{\mathrm{x}}$, the correlation energy functional has a diagrammatic expansion up to second order. Higher order terms are simulating higher order exchange diagrams.

correlation energies evaluated using the fully frequencydependent EXX kernel (TDEXX) are compared to those obtained by using the static (=adiabatic) kernel (AEXX) and the difference is seen to be relatively small. Notice that in the case of He the static kernel is the full kernel. These results have encouraged us to evaluate the correlation potentials also using the adiabatic approximation.

\section{CORRELATION ENERGIES AND POTENTIALS}

When evaluating the correlation energy for a system it is natural to use the self-consistent density for that particular system. From now on, the self-consistent density corresponding to the new functional in Eq. (8) is referred to as the RPAX density. Due to the stationary property of the total energy we expect, however, that evaluating correlation energies at a slightly different density will give almost the same result. ${ }^{15}$ This is, indeed, the case as can be seen in Table I, which also seems to demonstrate that the total energy is a minimum at the RPAX density. Clearly, the EXX kernel gives a large improvement over the too large RPA values. It also improves the MP2 results, which are here the self-consistent results given in Ref. 16. The latter approximation also follows from Eq. (1) if $\chi^{\lambda}$ is replaced by $\chi^{\lambda} \approx \chi_{s}+\lambda \chi_{s}\left[v+f_{\mathrm{x}}\right] \chi_{s}$, or if the logaritm in Eq. (8) is expanded to second order. The use of the adiabatic approximation is seen to be less severe yielding energies of the same quality as those obtained with the frequency-dependent kernel. As noticed before, the values in TDEXX, or in AEXX for that matter, are very accurate for these systems.

The $\mathrm{XC}$ potential is interesting in its own right. The highest occupied eigenvalue of the KS system exactly corresponds to the ionization potential ${ }^{18}$ and the larger part of the particle-conserving excitation energies consists of KS eigenvalue differences. ${ }^{19}$ In Tab. II we present ionization potentials produced by different KS potentials (RPAX, RPA, MP2 and EXX). As noticed previously, the RPA values improve over EXX and are also better than the MP2 values. Here, we see that the RPAX potential yields an even further improvement giving excellent ionization potentials for all atoms. In Fig. 2 we plot the same correlation potentials for $\mathrm{He}, \mathrm{Be}$, and Ne. The He RPAX potential almost coincide with the exact potential ${ }^{11}$ and the RPAX potential for Be is much closer to the exact than any other approximation we have tried - especially 
TABLE I: Correlation energies from a few different approximations. For a consistent comparison with CI results the correlation energy is here defined as the difference between the total energy and the Hartree-Fock energy. The fourth decimal is shown in parentheses in order to compare different approximations. (a.u.)

\begin{tabular}{c|llll|l|l|l}
\hline \hline$f_{\mathrm{xc}}:$ & TDEXX & AEXX & TDEXX & AEXX & RPA & $\mathrm{MP2}^{b}$ & $\mathrm{CI}^{c}$ \\
$v_{\mathrm{xc}}:$ & EXX & EXX & RPAX & RPAX & RPA & MP2 & \\
\hline $\mathrm{He}$ & $0.044(5)$ & $0.044(5)$ & $0.044(6)$ & $0.044(6)$ & 0.083 & 0.047 & 0.0420 \\
$\mathrm{Be}$ & $0.102(0)$ & $0.101(7)$ & $0.103(3)$ & $0.102(8)$ & 0.181 & 0.124 & 0.0943 \\
$\mathrm{Ne}$ & $0.388(9)$ & $0.377(1)$ & $0.390(3)$ & $0.377(8)$ & 0.596 & 0.480 & 0.3905 \\
$\mathrm{Mg}$ & $0.445(2)$ & $0.437(2)$ & $0.446(6)$ & $0.438(4)$ & 0.681 & 0.514 & 0.4383 \\
$\mathrm{Ar}$ & $0.727(8)$ & $0.710(6)$ & $0.728(7)$ & $0.711(2)$ & 1.091 & 0.844 & 0.7225 \\
\hline \hline
\end{tabular}

${ }^{b}$ From Ref. 16.

${ }^{c}$ From Ref. 17.

in the outer region. The Ne potential is also very accurate, yielding even a qualitative improvement by better describing the 2 s-shell giving rise to an extra structure in the RPAX, and the exact, correlation potential.

\section{STATIC POLARIZABILITIES AND VAN DER WAALS COEFFICIENTS}

The static polarizability is defined according to the formula

$$
\alpha(0)=-\int z \chi\left(\mathbf{r}, \mathbf{r}^{\prime}, \omega=0\right) z^{\prime} d \mathbf{r} d \mathbf{r}^{\prime},
$$

and the van der Waals coefficient, or $C_{6}$-coefficient, between ion $A$ and $B$ is given by

$$
C_{6}=\frac{3}{\pi} \int_{0}^{\infty} \alpha_{A}(i \omega) \alpha_{B}(i \omega) d \omega
$$

where $\alpha_{A}(i \omega)$ is the dynamic polarizability of ion $A$ calculated at imaginary frequencies. In previous work these quantities were calculated using the response function of the TDEXX approximation. It was then a natural choice to use the corresponding self-consistent EXX density in the evaluation. We then found, not so surprisingly, that our results closely resembled those of the TDHF approximation and were, therefore, not overly impressive. We

TABLE II: Ionization potentials obtained from the highest occupied KS eigenvalue of different KS potentials. (a.u.)

\begin{tabular}{llllll}
\hline \hline Atom & EXX & MP2 & RPA & RPAX & Exp. \\
\hline $\mathrm{He}$ & 0.918 & 0.893 & 0.902 & 0.904 & 0.904 \\
$\mathrm{Be}$ & 0.309 & 0.357 & 0.354 & 0.340 & 0.343 \\
$\mathrm{Ne}$ & 0.851 & 0.657 & 0.796 & 0.787 & 0.792 \\
$\mathrm{Mg}$ & 0.253 & 0.302 & 0.297 & 0.282 & 0.281 \\
$\mathrm{Ar}$ & 0.591 & 0.558 & 0.590 & 0.577 & 0.579 \\
\hline \hline
\end{tabular}

interpreted this partial failure to be a consequence of a rather poor description of the ground state. We found, however, also that the results are rather sensitive to the density used in the evaluation. In the present work we have instead evaluated the same EXX formula for the polarizability using the correlated density produced by our new correlation functional. The van der Waals energy is a pure correlation effect and one can argue that it should be evaluated at a correlated density and not just the exchange density like, e.g., the RPA or the RPAX density. This turns out to have rather drastic effect on the actual values moving them much closer to the more accurate values found in the literature as seen in Tab. III and IV. A clear improvement is found when correlated densities are used in the evaluation with a slight edge for our new RPAX density. It is also noticed that when calculating $C_{6}$-coefficients the adiabatic approximation is sufficient as also observed previously. ${ }^{24}$

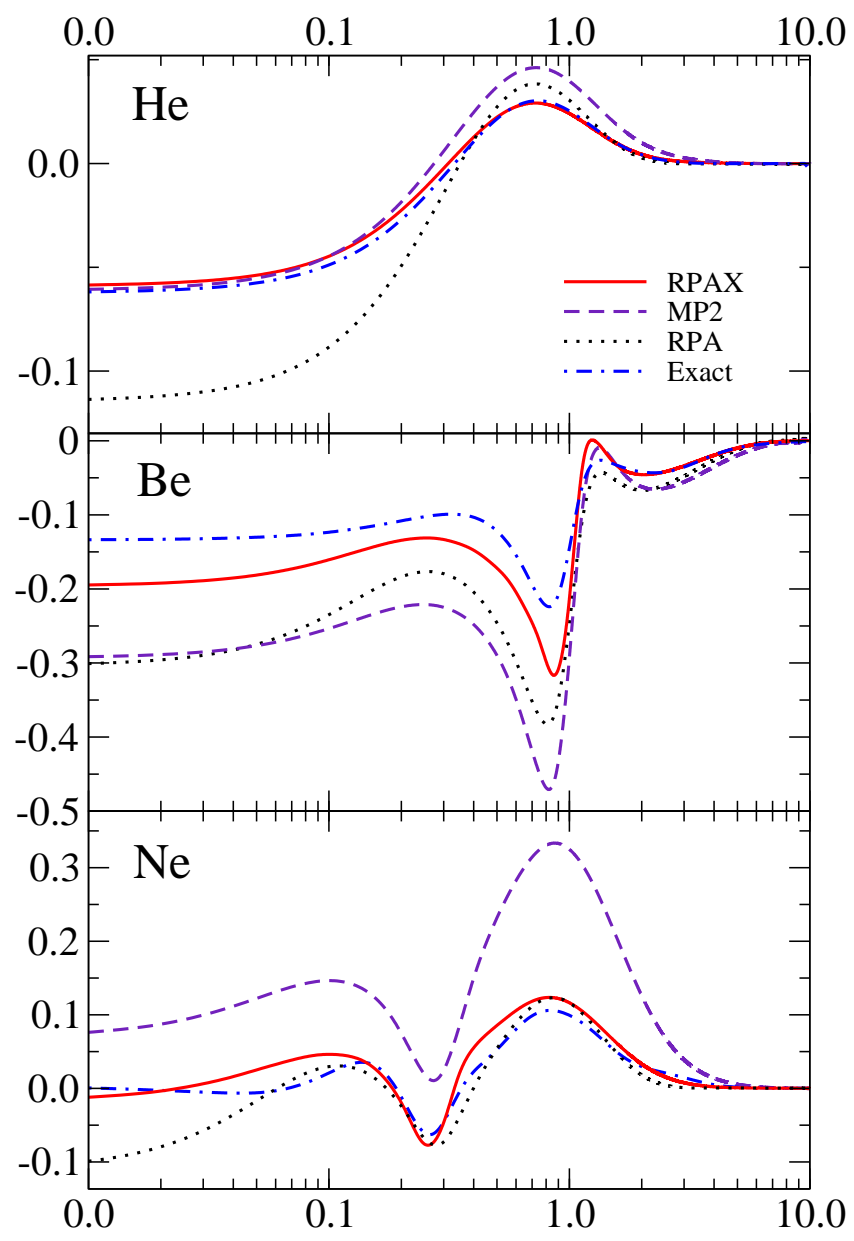

FIG. 2: Self-consistent correlation potentials for $\mathrm{He}, \mathrm{Be}$ and Ne in different approximations. The MP2 potential for $\mathrm{Be}$ is evaluated at the EXX density due to a variational instability. ${ }^{16}$ The exact potentials are those of Umrigar et al. $^{11}$ 
TABLE III: Static polarizabilities calculated from $\chi$ in the RPA and the TDEXX approximation. The latter has been evaluated using different potentials (EXX, RPA, RPAX, and Exact). (a.u.)

\begin{tabular}{c|cccc|c|c}
\hline \hline$f_{\text {xc }}:$ & \multicolumn{2}{|c|}{ TDEXX } & & RPA & Litt. \\
$v_{\text {xc }}$ & EXX & RPA & RPAX & Exact & RPA & \\
\hline $\mathrm{He}$ & 1.322 & 1.351 & 1.348 & 1.349 & 1.225 & $1.38^{a}$ \\
$\mathrm{Ne}$ & 2.372 & 2.577 & 2.613 & 2.555 & 2.424 & $2.67^{a}$ \\
$\mathrm{Ar}$ & 10.74 & 10.69 & 10.94 & - & 9.839 & $11.08^{a}$ \\
$\mathrm{Be}$ & 45.64 & 40.09 & 41.04 & 40.49 & 28.99 & $37.7^{b}$ \\
$\mathrm{Mg}$ & 81.66 & 70.37 & 71.67 & - & 51.56 & $71.35^{b}$ \\
\hline \hline
\end{tabular}

${ }^{a}$ From Ref. ${ }^{20}$

${ }^{b}$ From Ref. ${ }^{21}$

TABLE IV: van der Waals or $C_{6}$ coefficients calculated from $\chi$ in the RPA, the AEXX and the TDEXX approximation. The latter has been evaluated using different potentials (EXX, RPA, RPAX, and Exact). (a.u.)

\begin{tabular}{c|cccc|c|c|c}
\hline \hline$f_{\text {xc }}:$ & TDEXX & & & AEXX & RPA & Litt. \\
$v_{\text {xc }}:$ & EXX & RPA & RPAX & Exact & RPAX & RPA & \\
\hline $\mathrm{He}$ & 1.375 & 1.414 & 1.411 & 1.411 & 1.411 & 1.206 & $1.458^{a}$ \\
$\mathrm{Ne}$ & 5.506 & 6.091 & 6.191 & 6.021 & 6.161 & 5.523 & $6.383^{a}$ \\
$\mathrm{Ar}$ & 61.88 & 61.27 & 63.19 & - & 63.11 & 53.69 & $64.3^{a}$ \\
$\mathrm{Be}$ & 282.8 & 226.7 & 235.3 & 231.5 & 236.5 & 142.0 & $214^{b}$ \\
$\mathrm{Mg}$ & 767.5 & 617.8 & 634.3 & - & 632.3 & 385.6 & $627^{b}$ \\
\hline \hline
\end{tabular}

${ }^{a}$ From Ref. 22.

${ }^{b}$ From Ref. 23.

\section{CONCLUSIONS AND OUTLOOK}

In the present work we have decided to temporarily part from our familiar, systematic and conserving way of constructing improved approximations to XC potentials and kernels within TDDFT in the linear regime. Instead, we have tested the first step in an iterative scheme originally proposed by Peuckert. The starting point has been the previously studied EXX approximation for the $\mathrm{XC}$ potential and corresponding kernel. The method is described in detail in the sections above. The rational for this deviation has been the extraordinary accurate potentials obtained from this approach. The potentials are indeed very close to the exact XC potentials of DFT where these are known. Calculated ionization potentials for all atoms are close to experimental results in accordance with the well known fact that the highest occupied DF eigenvalue should equal minus the ionization potential.

We have in previous work calculated static polarizabilities, low lying particle-hole excitation energies, and van der Waals coefficients from the EXX approximation. These turned out to be very similar to those of TDHF theory and not very accurate. We argued then that these less satisfactory results were a consequence of a rather poor description of the ground state within the EXX approximation. Using instead, our new potentials to recalculate the mentioned properties results are in excellent agreement with experiment for all atoms studied.

We can thus state with confidence that we now have at our disposal an affordable and not too complicated way of obtaining accurate results for a large number of static and low-frequency dynamic properties of physical systems. And all this within a relative minor modification of the EXX approximation within TDDFT.

Of course, we have, at this stage, no idea about the possible conserving properties of the proposed scheme. This is an interesting project for future research. But, on the other hand, conservation laws and sum rules might not be of such great importance to the properties discussed here.

We should also mention that the results obtained here rely on the assumption that one can neglect the density and the frequency dependence of the $\mathrm{XC}$ kernel of the EXX. Based on the fact that this is no assumption in the case of a two-electron system we believe in the validity of this assumption but this must, of course, be further investigated. In the meanwhile we propose to use the new method as an effective tool for calculating physical properties within the realm of TDDFT.

\section{Acknowledgments}

This work was supported by the European Theoretical Spectroscopy Facility (INFRA-2007-211956). 
1 E. K. U. Gross and W. Kohn, Phys. Rev. Lett. 55, 2850 (1985).

2 M. Hellgren and U. von Barth, Phys. Rev. B 76, 075107 (2007).

3 M. Hellgren and U. von Barth, Phys. Rev. B 78, 115107 (2008).

4 M. Hellgren and U. von Barth, J. Chem. Phys. 131, 044110 (2009).

5 S. Kurth and U. von Barth, unpublished.

6 U. von Barth, N. E. Dahlen, R. van Leeuwen, and G. Stefanucci, Phys. Rev. B 72, 235109 (2005).

7 C.-O. Almbladh, U. von Barth, and R. van Leeuwen, Int. J. Mod. Phys. B 13, 535 (1999).

8 V. Peuckert, J. Phys. C-Solid State Physics 11, 4945 (1978).

9 D. C. Langreth and J. P. Perdew, Solid State Comm. 17, 1425 (1975).

10 C.-O. Almbladh, unpublished.

11 C. J. Umrigar and X. Gonze, Phys. Rev. A 50, 3827 (1994).

12 M. A. L. Marques, C. A. Ullrich, F. Nogueira, A. Rubio, K. Burke, and E. K. U. Gross, eds., Time-Dependent Density Functional Theory (Springer, Berlin, Heidelberg, 2006).

13 L. J. Sham and M. Schlüter, Phys. Rev. Lett. 51, 1888
(1983).

14 M. Grüning, A. Marini, and A. Rubio, J. Chem. Phys. 124, 154108 (2006).

15 We notice that the presented correlation energies are not obtained by just evaluating Eq. (8) at different densities but are calculated as the total density functional energy minus the exact HF energy.

16 H. Jiang and E. Engel, J. Chem. Phys. 123, 224102 (2005).

17 S. J. Chakravorty, S. R. Gwaltney, E. R. Davidson, F. A. Parpia, and C. F. Fischer, Phys. Rev. A 47, 3649 (1993).

18 C.-O. Almbladh and U. von Barth, Phys. Rev. B 31, 3231 (1985).

19 M. Petersilka, U. J. Gossmann, and E. K. U. Gross, Phys. Rev. Lett. 76, 1212 (1996).

20 S. Hirata, S. Ivanov, R. J. Bartlett, and I. Grabowski, Phys. Rev. A 71, 032507 (2005).

21 J. Mitroy and M. W. J. Bromley, Phys. Rev. A 68, 052714 (2003).

22 A. Kumar and W. J. Meath, Mol. Phys. 54, 823 (1985).

23 S. G. Porsev and A. Derevianko, Phys. Rev. A 65, 020701 (2002).

24 Y. Shigeta, K. Hirao, and S. Hirata, Phys. Rev. A 73, 010502 (2006). 